\title{
Comparison of Fragmentation and Dusting Laser Modes for Laser Ureteroscopy Lithotripsy of Ureteral Stones at a Single Center
}

\section{Bo-Han Chen}

MacKay Memorial Hospital

Tsu-Feng Lin ( $\nabla$ uro4272@gmail.com )

MacKay Memorial Hospital

\section{Marcelo Chen}

MacKay Memorial Hospital

Allen W. Chiu

MacKay Memorial Hospital

\section{Research Article}

Keywords: Dusting, Energy, Fragmentation, Frequency, Laser lithotripsy

Posted Date: December 29th, 2021

DOI: https://doi.org/10.21203/rs.3.rs-1167154/v1

License: (c) (i) This work is licensed under a Creative Commons Attribution 4.0 International License. Read Full License 


\section{Abstract}

\section{Background}

Laser ureteroscopic lithotripsy (URSL) is an efficacious treatment for ureteral stones. There have been few previous studies compared the different energy and frequency settings for URSL in a single center. The fragmentation and dusting laser mode were simultaneously used in our medical center. We compared the efficacy and outcomes of these two laser modalities for the treatment of ureteral stones.

\section{Methods}

Patients who underwent fragmentation or dusting laser URSL for ureteral stones were retrospectively reviewed. The demographic data, stone parameters, perioperative data and stone-free rates were analyzed between the two groups.

\section{Results}

There were a total of 421 patients with ureteral stones who met the study criteria. More patients in the dusting group had multiple ureteral stones and pyuria than in the fragmentation group. The fragmentation group had a better stone free rate and a lower push back rate compared with the dusting group. ( $82 \%$ vs. $71 \% ; 10 \%$ vs. $20 \%$ respectively, both $p<0.05$ ). Multivariate analysis revealed that stone basket use (odds ratio $[O R]=3.026 ; p<0.001$ ) significantly improved the stone free rate, whereas multiple stones $(O R=0.322 ; p<0.001)$, upper ureteral stone location $(O R=0.098 ; p=0.002)$ and pyuria $(O R=0.428$; $p=0.001)$ significantly decreased the stone free rate. The laser mode used was not significantly related to the stone free rate in the multivariate analysis.

\section{Conclusions}

Both laser modes were effective and safe for ureteral lithotripsy. Risk factors associated with a lower stone free rate were multiple stones, pyuria, upper ureteral stone location and an operation without the use of a stone basket.

\section{Background}

Ureteral stones and the symptoms related to ureteral obstruction, usually adversely affect the patients' quality of life. [1] There are several therapeutic options for ureteral stones, including observation and medical expulsive therapy, shock-wave lithotripsy, ureteroscopic lithotripsy, open surgery, laparoscopic stone removal and percutaneous antegrade ureteroscopy.[2, 3] The decision of which method is most suitable for each patient depends on the stone's anatomical location and characteristics, and the safety profile of each technique.[4] Since the development of the semirigid ureteroscope with a smaller caliber, ureteroscopic lithotripsy (URSL) has become an efficacious modality for the treatment of stones in all locations in the ureter.[2] 
Various different energy sources can be used for lithotripsy during a ureteroscopy, including pneumatic, ultrasound, electrohydraulic and laser.[3,5] Nowadays, holmium laser lithotripsy is one of the most widely used approaches by urologists because it leads to fewer complications and has a lower incidence of upward stone migration.[6, 7]

The holmium laser breaks down the stone via the power which is generated from a large crystal that is coupled to fibers.[8] The power can be adjusted by the parameters of energy, frequency and pulse width. [8] The high energy, low frequency setting is defined as the fragmentation mode, while the low energy, high frequency setting is defined as the dusting mode. $[8,9]$ The main advantage of dusting is that it produces smaller fragments and results in a shorter operation (OP) time; however, it requires a more advanced laser system which usually requires more expensive equipment.[8] Some studies thought that using the dusting laser mode could reduce the risk of ureteral injury, however, there is little evidence that one approach is better than the other.[9]

Previous studies have compared the efficacy and safety of different energy and frequency settings, but there have been few studies comparing the use of fragmentation and dusting laser systems in a single center. $[10,11]$ The fragmentation laser mode and the dusting laser mode were simultaneously used in our medical center, MacKay Memorial Hospital. In this study, we compared the efficacy and outcomes of these two laser modalities for the treatment of ureteral stones.

\section{Methods}

Patients aged $\geq 20$ years who underwent URSL for ureteral stones using a laser system in Mackay Memorial Hospital between September 2018 and June 2020, were retrospectively reviewed. Patients with radiolucent stones, those who were lost to follow-up, and those who had incomplete image records (a lack of either preoperative images or images within 1 postoperative month) were excluded. Patients who underwent concurrent retrograde intrarenal surgery (RIRS) or combination therapy with other treatment modalities were also excluded (Figure 1). This study was approved by the Institutional Review Board of Mackay Memorial Hospital as a retrospective observational cohort study.

The patients' age, gender, anesthesia method, admission rate, stone burden, stone number, stone laterality, stone location, ureteral condition, prevalence of pyuria, OP time, lithotripsy effectiveness, stone free rate, presence of push back, secondary intervention (extracorporeal shock wave (ESWL) or URSL), and complications, including ureteral injury and postoperative ureteral stricture rate, were recorded. The type of ureteral stent inserted was also recorded, including retrograde ureteral catheter (RUC; whistle tip ureteral catheter, Cook Medical, Bloomington, United States of America), double J (DJ; Universa soft, Cook Medical, Bloomington, United States of America) or stone basket (Zero Tip 2.4F, Boston Scientific, Marlborough, United States of America).

Depending on the patients' condition and the attending physicians' clinical decision, the patients were either postoperatively admitted to the urology department or they recovered from their URSL at an outpatient clinic. The anesthesia method was determined by the anesthesiologist following their 
preoperative evaluation. Stone location and number of stones were determined from a preoperative plain radiography of the kidney, ureter, and bladder (KUB). Stone burden was defined as the area of the stone calculated by our imaging system after the stone was delineated (Figure 2). Lithotripsy effectiveness was defined as the stone burden divided by the OP time ( $\mathrm{mm}^{2} /$ minutes). Pyuria was defined as $>10$ white blood cell counts in the preoperative urine analysis under a high power field. The stone's upward push back, ureter condition and postoperative injury were documented according to the surgeons' intraoperative findings. Follow-up plain radiography KUB or computerized tomography (CT) were performed within 1 month of the URSL. The definition of stone free is not standardized and differs greatly in previous literature.[9] In the current study, stone-free was defined as no residual stones $>3 \mathrm{~mm}$ in the ureter of the affected side within 1 postoperative month.[12] We searched the medical records for any complications and secondary interventions, including a repeat URSL, ESWL or RIRS for stones that remained in the ureter or were pushed back to the renal calyx during the initial operation.

A semirigid 6/7.5-F ureteroscope (Wolf, Knittlingen, Germany) was used for all procedures. All URSL operations were performed by the same experienced urology team for both modes. The initial settings for the fragmentation laser system (Auriga XL 50W, Boston Scientific, Marlborough, United States of America) with a $365 \mu \mathrm{m}$ fiber were: energy 0.8 Joules and frequency $8 \mathrm{Hertz}$. The initial settings for the dusting laser system (VersaPulse 60W, Lumenis, Yokneam, Israel) with a $365 \mu \mathrm{m}$ fiber were: energy 0.4 Joules and frequency $40 \mathrm{Hertz}$. Mild adjustments could be made depending on the surgeon. The patient was placed in a reverse Trendelenburg position and the intraluminal water pressure was decreased by lowering the water bottle. After the lithotripsy was finished, the larger stone fragments were removed using a retrieval basket and the smaller ones were left for spontaneous passage. At the end of the procedure, a ureteral stent was considered, depending on the fragmentation status of the residual stones, the ureteral condition, bleeding, and the index of suspicion for postoperative ureteral stricture. Perioperative intravenous broad spectrum antibiotics were given to all patients and oral antibiotics were given to outpatients postoperatively.

A t-test, a Chi-squared test and Fisher's exact test were used for statistical analysis, and the statistical significance was set at $p<0.05$. SPSS for Windows (version 21.0; IBM SPSS Statistics, IBM Corporation, Chicago, IL, USA) was used to perform the logistic regression analysis of risk factors for stone free rate. The t-test, Chi-squared test and Fisher's exact test were used to explore the differences between the fragmentation and dusting groups. All tests were two-sided, and $p$ values $<0.05$ were considered to be statistically significant.

\section{Results}

A total of 421 patients were analyzed, including 271 who received dusting laser URSL and 150 who received fragmentation laser URSL. The mean age was $54.1 \pm 12.63$ years in the dusting group and 53.9 \pm 13.57 years in the fragmentation group. The mean stone burden was $56.8 \pm 60.71 \mathrm{~mm}^{2}$ in the dusting group and $48.9 \pm 59.23 \mathrm{~mm}^{2}$ in the fragmentation group. There were no significant differences in gender, age, admission rate, anesthesia methods and stone laterality between the two groups. However, 
significantly more patients in the dusting group had multiple ureteral stones $(\mathrm{p}=0.023)$ and pyuria $(p<0.001)$ compared with the fragmentation group. There were no significant differences in stone location and ureteral condition between the two groups (Table 1).

Table 1

Patient characteristics compared between the dusting and fragmentation groups.

\begin{tabular}{|c|c|c|c|c|c|}
\hline \multirow[t]{2}{*}{ Total number } & \multicolumn{2}{|l|}{271} & \multicolumn{3}{|l|}{150} \\
\hline & Dusting & $\%$ & Fragmentation & $\%$ & $P$ value \\
\hline Female/Male & $114 / 157$ & $F: 42.1 \%$ & $50 / 100$ & $\mathrm{~F}: 33.3 \%$ & 0.078 \\
\hline Left/Right & $151 / 120$ & L: $55.7 \%$ & $84 / 66$ & L: $56 \%$ & 0.956 \\
\hline Admission rate, $\mathrm{n}(\%)$ & 206 & $76 \%$ & 125 & $83 \%$ & 0.079 \\
\hline Mean age (years) & 54.1 & SD:12.63 & 53.9 & SD: 13.57 & 0.89 \\
\hline Mean stone burden $\left(\mathrm{mm}^{2}\right)$ & 56.8 & SD: 60.71 & 48.9 & SD: 59.23 & 0.199 \\
\hline Anethesia & & & & & 0.312 \\
\hline Spinal & 102 & $38 \%$ & 64 & $43 \%$ & \\
\hline General & 169 & $62 \%$ & 86 & $57 \%$ & \\
\hline Number of stones, $\mathrm{n}(\%)$ & & & & & 0.023 \\
\hline Single & 219 & $81 \%$ & 134 & $89 \%$ & \\
\hline Multiple & 52 & $19 \%$ & 16 & $11 \%$ & \\
\hline \multicolumn{6}{|l|}{ Stone location } \\
\hline Upper & 234 & $86 \%$ & 135 & $90 \%$ & 0.275 \\
\hline Middle & 25 & $9 \%$ & 12 & $8 \%$ & 0.671 \\
\hline Lower & 20 & $7 \%$ & 10 & $7 \%$ & 0.785 \\
\hline \multicolumn{6}{|l|}{ Ureter condition, n (\%) } \\
\hline polyposis & 55 & $20 \%$ & 22 & $15 \%$ & 0.153 \\
\hline angulation & 40 & $15 \%$ & 26 & $17 \%$ & 0.487 \\
\hline stricture & 32 & $12 \%$ & 23 & $15 \%$ & 0.304 \\
\hline edema & 22 & $8 \%$ & 5 & $3 \%$ & 0.055 \\
\hline Pyuria, n (\%) & 110 & $41 \%$ & 33 & $22 \%$ & $<0.001$ \\
\hline
\end{tabular}


The treatment results revealed that the fragmentation group had a significantly higher stone free rate $(p=0.012)$ and a lower stone push back rate $(p=0.011)$ compared with the dusting group. The dusting group had a slightly higher lithotripsy effectiveness $(p=0.76)$ and postoperative ureteral stricture rate $(p=0.055)$ compared with the fragmentation group, but the differences were not statistically significant. There were no statistically significant differences in the OP time, ureteral stent insertion rate, stone basket usage rate, secondary intervention rate, ureteral injury rate and complication rate between the two groups (all p>0.05) (Table 2).

Table 2

Comparison of outcomes for lithotripsy between the dusting and fragmentation groups.

\begin{tabular}{|c|c|c|c|c|c|}
\hline \multirow[t]{2}{*}{ Total number } & \multicolumn{2}{|l|}{271} & \multicolumn{3}{|l|}{150} \\
\hline & Dusting & $\%$ & Fragmentation & $\%$ & $P$ value \\
\hline OP time (min) & 38.2 & SD: 17.82 & 37.6 & SD: 19.14 & 0.752 \\
\hline Effectiveness (mm2/min) & 1.49 & & 1.45 & & 0.76 \\
\hline Ureteral stent insertion rate, $\mathrm{n}(\%)$ & 231 & $85 \%$ & 127 & $85 \%$ & 0.875 \\
\hline RUC & 12 & $4 \%$ & 9 & $6 \%$ & 0.478 \\
\hline DJ & 219 & $81 \%$ & 118 & $79 \%$ & 0.598 \\
\hline Basket use, n (\%) & 161 & $59 \%$ & 93 & $62 \%$ & 0.603 \\
\hline Stone free rate, $n(\%)$ & 192 & $71 \%$ & 123 & $82 \%$ & 0.012 \\
\hline Push back rate, $\mathrm{n}(\%)$ & 53 & $20 \%$ & 15 & $10 \%$ & 0.011 \\
\hline Secondary intervention rate, $\mathrm{n}(\%)$ & 50 & $18 \%$ & 29 & $19 \%$ & 0.824 \\
\hline Ureter injury, n (\%) & 0 & $0 \%$ & 1 & $1 \%$ & 0.356 \\
\hline Complication, n (\%) & 11 & $4 \%$ & 3 & $2 \%$ & 0.281 \\
\hline Postoperative ureter stricture, $\mathrm{n}(\%)$ & 9 & $3 \%$ & 0 & $0 \%$ & 0.03 \\
\hline
\end{tabular}

Complications included three cases of bleeding or oozing during the OP, one case of postoperative prostatitis, one case of leg dropping from the lithotomy position and nine cases of postoperative ureteral strictures. The three patients with intraoperative bleeding or oozing had a prolonged OP time for a hemostasis procedure. The patient who suffered from prostatitis was diagnosed by CT image, admitted again 5 days after the URSL and received intravenous antibiotics for 10 days. The patient with one leg dropping from the lithotomy position received fluoroscopic imaging and there was no fracture or dislocation. Among the nine patients with postoperative ureteral stricture, two of them had laser ureterotomy, four of them had DJ re-insertion for 1 to 1.5 months and three of them did not need further treatment. 
Univariate analysis showed that patients who were stone free were significantly less likely to have received the dusting laser system (odds ratio $[O R]=0.533, p=0.012$ ), and had a significantly higher stone basket usage rate $(O R=2.82,<0.001)$, fewer multiple stones $(O R=0.375, p<0.001)$, fewer upper ureteral stones $(O R=0.102, p=0.002)$ and less pyuria $(O R=0.34, p<0.001)$ compared with patients who were not stone free (Table 3). Multivariate logistic analysis revealed that patients who were stone free had a significantly higher stone basket usage rate $(O R=3.026, p<0.001)$, fewer multiple stones $(O R=0.322$, $p<0.001)$, fewer upper ureteral stones $(O R=0.098, p=0.002)$ and less pyuria $(O R=0.428, p=0.001)$ compared with those patients who were not stone free. The dusting laser system had a slightly lower stone free rate, however, the multivariate logistic analysis did not reveal a statistically significant difference (Table 3).

Table 3

Univariate and multivariate logistic regression analysis of the stone free rate.

\begin{tabular}{|c|c|c|c|c|c|c|c|c|}
\hline \multirow{3}{*}{ Dusting system } & \multicolumn{4}{|c|}{ Univariate } & \multicolumn{4}{|c|}{ Multivariate } \\
\hline & \multirow{2}{*}{$\begin{array}{l}p \\
0.012\end{array}$} & \multirow{2}{*}{$\begin{array}{l}\text { OR } \\
0.533\end{array}$} & \multicolumn{2}{|c|}{$95 \% \mathrm{Cl}$ of OR } & \multirow{2}{*}{$\begin{array}{l}\mathbf{p} \\
0.063\end{array}$} & \multirow{2}{*}{$\begin{array}{l}\text { OR } \\
0.6\end{array}$} & \multicolumn{2}{|c|}{$95 \% \mathrm{Cl}$ of OR } \\
\hline & & & 0.326 & 0.873 & & & 0.35 & 1.028 \\
\hline Basket use & $<0.001$ & 2.82 & 1.79 & 4.43 & $<0.001$ & 3.026 & 1.844 & 4.966 \\
\hline General anethesia & 0.462 & 1.183 & 0.756 & 1.85 & & & & \\
\hline Gender (female/male) & 0.871 & 0.963 & 0.614 & 1.511 & & & & \\
\hline Admission & 0.467 & 0.814 & 0.468 & 1.417 & & & & \\
\hline Laterality (left/right) & 0.851 & 0.958 & 0.615 & 1.494 & & & & \\
\hline Multiple stones & $<0.001$ & 0.375 & 0.218 & 0.646 & $<0.001$ & 0.322 & 0.175 & 0.591 \\
\hline Upper ureteral stone & 0.002 & 0.102 & 0.024 & 0.427 & 0.002 & 0.098 & 0.022 & 0.433 \\
\hline \multicolumn{9}{|l|}{ Ureteral condition } \\
\hline Polyposis & 0.066 & 1.824 & 0.96 & 3.466 & & & & \\
\hline Angulation & 0.051 & 0.57 & 0.325 & 1.002 & & & & \\
\hline Stricture & 0.778 & 1.101 & 0.566 & 2.14 & & & & \\
\hline Edema & 0.582 & 0.786 & 0.334 & 1.853 & & & & \\
\hline Pyuria & $<0.001$ & 0.341 & 0.216 & 0.537 & 0.001 & 0.428 & 0.262 & 0.698 \\
\hline
\end{tabular}

\section{Discussion}


Due to advances in technology, there are now a wide variety of power generators that can be used for ureteroscopic lithotripsy. In addition to pneumatic lithotripsy, laser lithotripsy has now become a predominant method for urinary stone treatment worldwide.[8] Pneumatic lithotripsy is thought to have a lower risk of ureteral perforation compared with laser lithotripsy, but it is associated with a higher rate of stone push back into the renal pelvis.[13] Chen et al. reported that laser lithotripsy is superior to pneumatic lithotripsy for the management of ureteral stones in terms of the stone free rate and the secondary intervention rate for stones of all sizes, at their institute.[12] Yin et al. also found that laser lithotripsy had advantages over pneumatic lithotripsy, with a high stone-free rate and a low migration rate in their meta-analysis. [6] If properly used, the laser generator provides a highly effective tool for disintegrating stones in the urinary tract with minimal additional safety concerns.[14]

Furthermore, there have been large improvements in the laser technique. For example, the Moses and vapor tunnel modulations have been designed, which help urologists to deal with stones in the urinary tract. Bozzini et al. found that the vapor tunnel technique significantly reduced retropulsion of the stone, the procedure time and ureteral lesions, and they thought that the vapor tunnel technique would be an excellent setting option for use with vulnerable ureteral walls. [15] Ibrahim et al. also found that Moses technology was associated with a significantly lower procedure time and less retropulsion in their doubleblinded prospective randomized trial. [16] The vapor tunnel technique had a significantly higher stone free rate compared with the regular settings, while the Moses technique showed a mild improvement. $[15,16]$ In our study, we found that the dusting mode had a significantly lower stone free rate in the univariate analysis compared with the fragmentation mode but there was no significant difference in the multivariate analysis. Therefore, we believe that both of these techniques are effective and safe for ureteral lithotripsy. However, because some of these technologies are relatively novel, more comprehensive studies may be needed to confirm the efficacy and safety of these innovations.

Among the different types of laser sources, holmium: yttrium-aluminum-garnet (Ho:YAG) is the most popular source for URSL because of its ability to fragment stones of any composition and its excellent safety profile.[16] The laser power of Ho:YAG can be highly absorbed in water and has a low penetration depth, therefore, Ho:YAG does little injury to the surrounding tissue.[8] The laser is generated and releases its energy from the tip of a fiber. The stones are then disintegrated into fragments or dust.[8] The pulse energy settings for the holmium laser usually range from 0.2 to 2.0 Joules and the frequency setting usually ranges from 4 to 80 Hertz depending on the manufacturer and the model of laser console used. [9] Different settings may cause diverse effects on the stones. The higher the pulse energy used, the more stone retropulsion occurs. Whereas, changes in frequency had a minimal effect on retropulsion when the energy and fiber diameter were kept constant.[17] In our study, the dusting group had a significantly lower stone free rate and a higher stone push back rate compared with the fragmentation group. However, in the multivariate analysis, the laser mode used was not significantly related to the stone free rate. The authors hypothesize that these findings could be due to the significant diversity of the patients' characteristics, especially pyuria and multiple stones, which may have hindered the URSL procedure and its outcomes. 
Although URSL has become a common surgery, its associated risk of urinary tract infection is about $25 \%$ in patients without prophylactic antibiotics.[18] To prevent patients from postoperative urosepsis, surgeons need to accomplish the URSL as quickly as possible, particularly for patients with pyuria.[19] If the patient is simultaneously suffering from a severe infection, the surgeon should break through the obstructive ureteral stone rapidly and place a ureteral stent for emergent drainage of the renal pelvis, instead of crushing the stone into smaller pieces. Under these circumstances the stone free rate can be decreased, and in the current study patients with pyuria had a significantly lower stone free rate. On the other hand, compared with solitary stones, multiple stones can also decrease the stone free rate in lithotripsy surgery.[20] In our study, multiple stones were also a significant risk factor for a lower stone free rate. Stone location also has a significant effect on stone clearance. Degirmenci et al. stated that the stone clearance rate was higher for lower ureteral stones compared with upper ureteral stones.[21] In our study, patients with upper ureteral stones showed a significantly lower stone free rate compared with stones in other locations. Furthermore, a review by Brain et al. found that using a stone basket was associated with a more complete initial stone clearance.[9] We also found similar results, as URSL with basket use had a higher stone free rate compared with URSL without basket use.

The current study had several limitations. First, this was a retrospective study, which means there was a patient selection bias, including some significant differences in the patient characteristics. Second, we did not set a standard follow-up duration for all of the patients. Our postoperative follow-up time depended on the patient's symptoms and their willingness to engage with clinicians. Asymptomatic patients tended to have a longer follow-up time compared with patients who suffered from immediate symptoms. Third, we did not do a complete analysis of the stone composition, which could have influenced the effectiveness of lithotripsy. However, in Asia, calcium oxalate is the most frequent component of stones and it accounts for about $90 \%$ of all calculi in the urinary tract.[22] Therefore, the bias caused by stone composition may not have a large effect. Fourth, we used two different machines for the two different laser settings. There may be more bias between the two machines than if the two different settings were used on the same machine. Finally, not all of the URSL in the present study were performed by the same urologist. However, all the urologists involved in this study had achieved the same qualification. In other words, there was only a little operating difference between each surgeon in the urologist team.

\section{Conclusions}

Both laser modes were effective and safe for ureteral lithotripsy. The energy and frequency settings did not have a significant effect on the stone free rate in these two modalities. Risk factors for a lower stone free rate included multiple stones $(\mathrm{OR}=0.322)$, pyuria $(\mathrm{OR}=0.428)$ and upper ureteral stone location $(\mathrm{OR}=0.098)$. Risk factors for a higher stone free rate were the use of a stone basket $(\mathrm{OR}=3.026)$.

\section{Abbreviations}

URSL: ureteroscopic lithotripsy 
OP: operation

ESWL: extracorporeal shock wave

RIRS: retrograde intrarenal surgery

RUC: retrograde ureteral catheter

DJ: double J stent

KUB: kidney, ureter, and bladder

CT: computerized tomography

Ho:YAG: Holmium: yttrium-aluminum-garnet

\section{Declarations}

\section{Ethics approval and consent to participate}

The present study, including its research protocols and data collection, were approved by the Institutional Review Board of Mackay Memorial Hospital. All methods were performed in accordance with the relevant guidelines and regulations and approved by the Institutional Review Board of Mackay Memorial Hospital. (IRB Number:21MMHIS242e)

\section{Consent for publication}

In view of the retrospective nature of the study, the need to obtain the patients' informed consent was waived by the Institutional Review Board of Mackay Memorial Hospital. (IRB Number:21MMHIS242e)

\section{Availability of data and materials}

Data and material pertaining to this study are in the patient's secure medical records in Mackay Memorial Hospital and are available from the corresponding author on reasonable request.

\section{Competing interests}

The authors declare no conflict of interest.

\section{Funding}




\section{Authors' contributions}

Conception and design, acquisition of data, analysis and interpretation of data, drafting of the manuscript, statistical analysis: BHC, TFL

Critical revision of the manuscript for important intellectual content, supervision: MC, AWC All authors reviewed the manuscript.

\section{Acknowledgements}

Not applicable.

\section{References}

1. Zhang LW, Fei X, Song Y: The clinical efficacy of novel vacuum suction ureteroscopic lithotripsy in the treatment of upper ureteral calculi. World Journal of Urology 2021.

2. Preminger GM, Tiselius H-G, Assimos DG, Alken P, Buck C, Gallucci M, Knoll T, Lingeman JE, Nakada SY, Pearle MS et al: 2007 Guideline for the Management of Ureteral Calculi. Journal of Urology 2007, 178(6):2418-2434.

3. Enikeev D, Grigoryan V, Fokin I, Morozov A, Taratkin M, Klimov R, Kozlov V, Gabdullina S, Glybochko $P$ : Endoscopic lithotripsy with a SuperPulsed thulium-fiber laser for ureteral stones: A single-center experience. International Journal of Urology 2021, 28(3):261-265.

4. Romeu G, Marzullo-Zucchet LJ, Díaz J, Villarroya S, Budía A, Ordaz DdG, Caballer V, Vivas D: Comparing extracorporeal shock wave lithotripsy and ureteroscopy laser lithotripsy for treatment of urinary stones smaller than $2 \mathrm{~cm}$ : a cost-utility analysis in the Spanish clinical setting. World Journal of Urology 2021.

5. Black KM, Aldoukhi AH, Teichman JMH, Majdalany SE, Hall TL, Roberts WW, Ghani KR: Pulse modulation with Moses technology improves popcorn laser lithotripsy. World Journal of Urology 2020.

6. Yin X, Tang Z, Yu B, Wang Y, Li Y, Yang Q, Tang W: Holmium: YAG laser lithotripsy versus pneumatic lithotripsy for treatment of distal ureteral calculi: a meta-analysis. J Endouro/ 2013, 27(4):408-414.

7. Rabani SM, Rabani S, Rashidi N: Laser Versus Pneumatic Lithotripsy With Semi-Rigid Ureteroscope; A Comparative Randomized Study. J Lasers Med Sci 2019, 10(3):185-188.

8. Aldoukhi AH, Black KM, Ghani KR: Emerging Laser Techniques for the Management of Stones. Urol Clin North Am 2019, 46(2):193-205. 
9. Matlaga BR, Chew B, Eisner B, Humphreys M, Knudsen B, Krambeck A, Lange D, Lipkin M, Miller NL, Monga $\mathrm{M}$ et al: Ureteroscopic Laser Lithotripsy: A Review of Dusting vs Fragmentation with Extraction. J Endouro/ 2018, 32(1):1-6.

10. Aldoukhi AH, Roberts WW, Hall TL, Teichman JMH, Ghani KR: Understanding the Popcorn Effect During Holmium Laser Lithotripsy for Dusting. Urology 2018, 122:52-57.

11. Sea J, Jonat LM, Chew BH, Qiu J, Wang B, Hoopman J, Milner T, Teichman JM: Optimal power settings for Holmium:YAG lithotripsy. J Urol 2012, 187(3):914-919.

12. Chen L-C, Chiu AW, Lin W-R, Lin W-C, Yang S, Hsu J-M, Chow Y-C, Tsai W-K, Chiang P-K, Chen M: Comparison of pneumatic and Holmium laser ureteroscopic lithotripsy for upper third ureteral stones. Urological Science 2017, 28(2):101-104.

13. Piergiovanni M, Desgrandchamps F, Cochand-Priollet B, Janssen T, Colomer S, Teillac P, Le Duc A: Ureteral and bladder lesions after ballistic, ultrasonic, electrohydraulic, or laser lithotripsy. J Endourol 1994, 8(4):293-299.

14. Vassar GJ, Chan KF, Teichman JM, Glickman RD, Weintraub ST, Pfefer TJ, Welch AJ: Holmium: YAG lithotripsy: photothermal mechanism. J Endourol 1999, 13(3):181-190.

15. Bozzini G, Berti L, Besana U, Calori A, Maltagliati M, Roche JB, Gözen AS, Breda A, Pini G, Pastore AL et al: "Vapor Tunnel": Advantages of a New Setting Option for Urgent Holmium Laser Lithotripsy with Cyber-Ho. Videourology 2020, 34(2).

16. Ibrahim A, Elhilali MM, Fahmy N, Carrier S, Andonian S: Double-Blinded Prospective Randomized Clinical Trial Comparing Regular and Moses Modes of Holmium Laser Lithotripsy. J Endourol 2020, 34(5):624-628.

17. White MD, Moran ME, Calvano CJ, Borhan-Manesh A, Mehlhaff BA: Evaluation of retropulsion caused by holmium:YAG laser with various power settings and fibers. $J$ Endouro/ 1998, 12(2):183-186.

18. Hsieh $\mathrm{CH}$, Yang SS, Lin CD, Chang SJ: Are prophylactic antibiotics necessary in patients with preoperative sterile urine undergoing ureterorenoscopic lithotripsy? BJU Int 2014, 113(2):275-280.

19. Bai T, Yu X, Qin C, Xu T, Shen H, Wang L, Liu X: Identification of Factors Associated with Postoperative Urosepsis after Ureteroscopy with Holmium: Yttrium-Aluminum-Garnet Laser Lithotripsy. Urol Int 2019, 103(3):311-317.

20. Ozgor F, Kucuktopcu O, Ucpinar B, Gurbuz ZG, Sarilar O, Berberoglu AY, Baykal M, Binbay M: Is There A Difference Between Presence of Single Stone And Multiple Stones in Flexible Ureterorenoscopy And Laser Lithotripsy For Renal Stone Burden < 300mm2 ? Int Braz J Urol 2016, 42(6):1168-1177.

21. Degirmenci T, Gunlusoy B, Kozacioglu Z, Arslan M, Kara C, Koras O, Minareci S: Outcomes of ureteroscopy for the management of impacted ureteral calculi with different localizations. Urology 2012, 80(4):811-815.

22. Liu Y, Chen Y, Liao B, Luo D, Wang K, Li H, Zeng G: Epidemiology of urolithiasis in Asia. Asian J Urol 2018, 5(4):205-214.

\section{Figures}




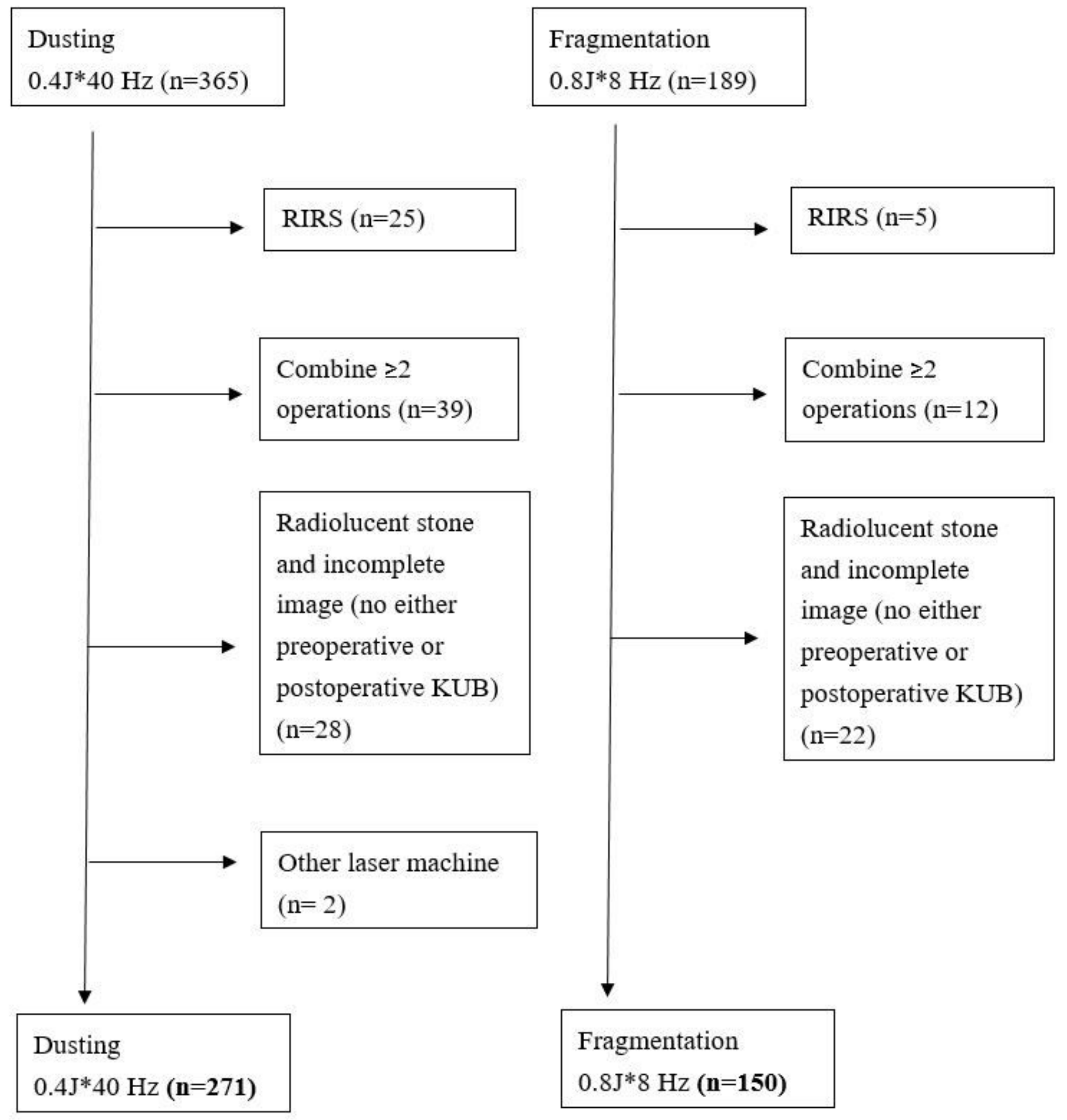

Figure 1

Flowchart of patient selection. 


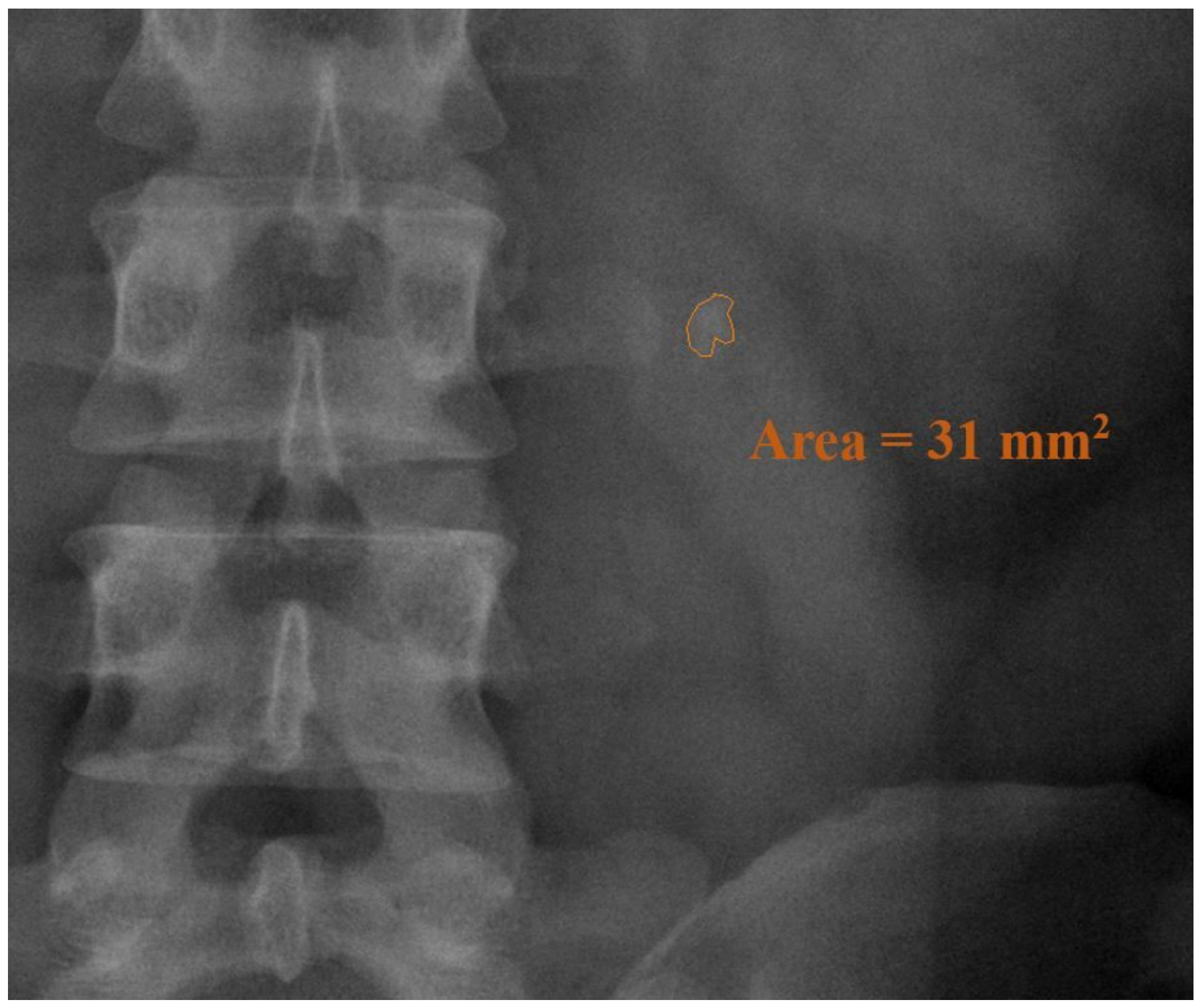

Figure 2

Area of the stone as calculated by the imaging system. 